\title{
ON THE QUENCHING RATE ESTIMATE
}

\author{
BY \\ JONG-SHENQ GUO \\ Institute of Applied Mathematics, National Tsing Hua University, Hsinchu, Taiwan
}

§1. Introduction. In this paper, we consider the problem

$$
\begin{aligned}
& u_{t}-u_{x x}=(1-u)^{-\beta}, \quad|x|<l, t>0, \\
& u( \pm l, t)=0, \quad t>0, \\
& u(x, 0)=u_{0}(x), \quad|x| \leq l,
\end{aligned}
$$

where $\beta>0, l>0,0 \leq u_{0}<1$ is smooth. The solution $u$ of $(1.1)$ is said to be quenching if $u$ reaches 1 in finite time $T$. Note that in this case $u_{t}$ blows up at the same time $T$. This phenomenon has been studied by many authors (see, for example, the references cited in [8] and [11]). In particular, for any $\beta>0$ there exists a positive constant $l_{*}=l_{*}(\beta)$ such that $u$ quenches for any $u_{0}$ if $l>l_{*}$. Hereafter we shall assume that $u$ quenches and that $u_{0}$ satisfies

$$
u_{0}^{\prime \prime}+\left(1-u_{0}\right)^{-\beta} \geq 0 \text {. }
$$

Let $T \in(0, \infty)$ be the quenching time for $u$. We say that $a$ is a quenching point for $u$ if there exists a sequence $\left\{\left(x_{n}, t_{n}\right)\right\}$ such that $x_{n} \rightarrow a, t_{n} \uparrow T$, and $u\left(x_{n}, t_{n}\right) \rightarrow 1$ as $n \rightarrow \infty$. The set of all such points (for the same $T$ ) is called the quenching set.

In [8] we first proved that the quenching set consists of finite points which remain a positive distance from $x= \pm l$. Then we studied the quenching rate of the solution near any quenching point. Let $\gamma=(\beta+1)^{-1}$ and $k=\gamma^{-\gamma}$. We obtained the following quenching rate estimate.

THEOREM A. If $a$ is a quenching point, then

$$
\lim _{t \uparrow T}(1-u(x, t))(T-t)^{-\gamma}=k
$$

uniformly for $|x-a| \leq C \sqrt{T-t}$ for any positive constant $C$.

But, there we only proved this theorem for $\beta \geq 3$. In [11], Levine conjectured that this theorem should hold for all $\beta>0$. Recently, Fila and Hulshof [3] improved this result to any $\beta \geq 1$ using a convexity argument of [5]. The purpose of this paper is to complete this quenching rate estimate for any $\beta>0$.

Received February 28, 1990.

Key words and phrases. Quenching, quenching point, quenching set, quenching rate, positively invariant region.

(C)1991 Brown University 
Recall the similarity variables

$$
\begin{gathered}
y=(x-a) / \sqrt{T-t}, \quad s=-\ln (T-t), \\
w(y, s)=(1-u(x, t))(T-t)^{-\gamma} .
\end{gathered}
$$

Then $w$ satisfies the equation

$$
w_{s}=w_{y y}-\frac{1}{2} y w_{y}+\gamma w-w^{-\beta}
$$

in the set $W=\{(y, s):|y \exp (-s / 2)+a|<l, s>-\ln T\}$. The key step for the proof of Theorem $\mathrm{A}$ is to show that the only positive global solution to

$$
w^{\prime \prime}-\frac{1}{2} y w^{\prime}+\gamma w-w^{-\beta}=0, \quad y \in \mathbf{R},
$$

under some growth condition at $|y|=\infty$, is the constant solution $w \equiv k$.

We note that the quenching rate estimate for the corresponding higher-dimensional radial case for $\beta>1$ was obtained by the author in [9]. Recently, Fila, Hulshof, and Quittner [4] have completed this result for all $\beta>0$.

This paper is organized as follows. In Sec. 2, we give the asymptotic behavior of solutions $w$ of (1.5) at $|y|=\infty$ by using the method of [9](see also [2]). Then we apply this result to obtain the quenching rate estimate (1.3) for $0<\beta<1$ in Sec. 3 .

$\S 2$. Asymptotic behaviors. In this section, we let $w=w(y)$ be any positive global solution of (1.5). The main result of this section is as follows.

THEOREM 2.1. If $w(y)$ is not identically equal to $k$, then $w(y)$ behaves either as $|y|^{2 \gamma}$ or as $|y|^{-(1+2 \gamma)} \exp \left(y^{2} / 4\right)$ at $|y|=\infty$.

Let

$$
f(w)=\gamma w-w^{-\beta} \text { and } F(w)=\int_{k}^{w} f(s) d s, \quad w>0 .
$$

Note that $f(w)>0$ if $w>k,<0$ if $w<k$; and $F(w)>0$ for $w \neq k$. Rewrite (1.5) as

$$
\left[w^{\prime 2} / 2+F(w)\right]^{\prime}(y)=y w^{\prime 2}(y) / 2 .
$$

Since the right-hand side of $(2.1)$ is nonnegative for $y \geq 0$, the limit

$$
\lim _{y \rightarrow \infty}\left[w^{\prime 2} / 2+F(w)\right](y)=l_{+}
$$

exists and is nonnegative. We claim that $l_{+}>0$ if $w \not \equiv k$. Indeed, if $l_{+}=0$, then $w^{\prime}(y) \rightarrow 0$ and $w(y) \rightarrow k$ as $y \rightarrow \infty$. By integrating (2.1) from $y$ to $\infty$, we obtain that $w^{\prime}(y) \equiv 0$ and $w \equiv k$ for $y \geq 0$. This contradiction leads to the conclusion $l_{+}>0$. Let $v=w^{\prime}$. Then $v^{\prime}=(y / 2) v-f(w)$.

Lemma 2.2. Let $\alpha$ be a positive constant. Then the region

$$
A_{\alpha} \equiv\{(w, v): w \geq k, v \geq \alpha w\}
$$

is a positively invariant region, i.e., there exists $y_{0}=y_{0}(\alpha)$ such that if $y_{1} \geq y_{0}$ and $\left(w\left(y_{1}\right), v\left(y_{1}\right)\right) \in A_{\alpha}$ then $(w(y), v(y)) \in A_{\alpha}, \forall y \geq y_{1}$.

Proof. Take $y_{0}=2(\alpha+\gamma / \alpha)$. Since the vector field $\left(w^{\prime}(y), v^{\prime}(y)\right)$ is always pointing inward to the region $A_{\alpha}$ for $y \geq y_{0}$, the lemma follows. 
Lemma 2.3. Suppose that $w(y) \not \equiv k$. Then $w$ cannot assume the value $k$ at infinitely many points as $y \rightarrow \infty$.

Proof. Suppose that there is a sequence $y_{m} \rightarrow \infty$ as $m \rightarrow \infty$ such that $w\left(y_{m}\right)=$ $k, \forall m$. Without loss of generality we may assume that $w^{\prime}\left(y_{m}\right)>0$ for all $m$. Recall that $l_{+}>0$. Hence

$$
w^{\prime}\left(y_{m}\right) \rightarrow \sqrt{2 l_{+}} \text {as } m \rightarrow \infty .
$$

Take any number $\alpha \in\left(0, \sqrt{2 l_{+}}\right)$and consider the positively invariant set $A=A_{\alpha / k}$. Let $y_{0}=y_{0}(\alpha / k)$ be the constant obtained in Lemma 2.2. Then there is an $m_{0}$ sufficiently large such that $w^{\prime}\left(y_{m_{0}}\right) \geq \alpha$ and $y_{m_{0}} \geq y_{0}$. Since $\left(w\left(y_{m_{0}}\right), v\left(y_{m_{0}}\right)\right) \in A$, from Lemma 2.2 it follows that $(w(y), v(y)) \in A$ for all $y \geq y_{m_{0}}$. Thus $v(y)>$ $0, \forall y \geq y_{m_{0}}$, which is a contradiction and the lemma is proved.

Let $w(y)$ be a nonconstant positive global solution of (1.5). From Lemma 2.3 it follows that either $w(y)>k, \forall y \geq \bar{y}$, or $w(y)<k, \forall y \geq \bar{y}$, for some $\bar{y}>0$. From the differential equation (1.5), we observe that any critical point $y$ of $w$ is a local maximum point if $w(y)>k$, and is a local minimum point if $w(y)<k$. Moreover, by the local uniqueness of solutions of ordinary differential equations, there cannot exist a point $y$ with $w(y)=k$ and $w^{\prime}(y)=0$ except when $w \equiv k$. Therefore, there is $y_{0} \geq \bar{y}$ such that either

$$
w^{\prime}(y)>0, \quad \forall y \geq y_{0}, \quad \text { or } \quad w^{\prime}(y)<0, \quad \forall y \geq y_{0} .
$$

Let

$$
L=\lim _{y \rightarrow \infty} w(y) .
$$

We claim that $L>k$, if $w^{\prime}(y)>0$ for all $y \geq y_{0}$. Indeed, if $L \leq k$, then from the equation

$$
w^{\prime \prime}=y w^{\prime} / 2-f(w)
$$

we obtain that $w^{\prime \prime}(y)>0$ for $y \geq y_{0}$. Thus

$$
w(y)=w\left(y_{0}\right)+\int_{y_{0}}^{y} w^{\prime}(\xi) d \xi \geq w^{\prime}\left(y_{0}\right)\left(y-y_{0}\right) \rightarrow \infty
$$

as $y \rightarrow \infty$, a contradiction. Hence $L>k$, if $w^{\prime}>0, \forall y \geq y_{0}$. Similarly, $L<k$, if $w^{\prime}(y)<0$ for all $y \geq y_{0}$.

Lemma 2.4. If $w \not \equiv k$, then $w$ must be strictly increasing to $+\infty$ as $y \rightarrow \infty$.

Proof. Suppose that $L<\infty$. Since the integral

$$
\int_{y_{0}}^{\infty} w^{\prime}(y) d y=L-w\left(y_{0}\right)
$$

is finite, there is a sequence $y_{m} \rightarrow \infty$ such that $w^{\prime}\left(y_{m}\right) \rightarrow 0$ as $m \rightarrow \infty$. Rewrite ..5) as

$$
w^{\prime \prime} / y-w^{\prime} / 2=-f(w) / y
$$


and integrate it from $y_{0}$ to $y_{m}$. Using an integration by parts for the first term, the integral on the left-hand side remains bounded as $m \rightarrow \infty$. But,

$$
\left|\int_{y_{0}}^{y_{m}} \frac{f(w(y))}{y} d y\right| \rightarrow \infty
$$

as $m \rightarrow \infty$. This contradiction leads to the conclusion of the lemma.

Proof of Theorem 2.1. From Lemma 2.4 we can easily obtain the asymptotic behavior of $w(y)$ at $y=\infty$ by the method used in [9]. The motivation is from Remark 2 in [2] and the proof is based on using L'Hôpital's rule (cf. [2] and [9]). For the reader's convenience, we outline the proof here.

First, using Lemma 2.2 and 2.4, we can show that the limit

$$
\alpha \equiv \lim _{y \rightarrow \infty} \frac{w^{\prime}(y)}{w(y)}
$$

exists and that $\alpha$ is either 0 or $\propto$. Suppose that $\alpha=0$. Then we have

$$
\lim _{y \rightarrow \infty} \frac{y w^{\prime}(y)}{w(y)}=2 \gamma,
$$

by applying L'Hôpital's rule and using the formula

$$
\frac{w^{\prime}(y)}{w(y)}=\exp \left(y^{2} / 4\right) \int_{y}^{\infty}[\gamma+a(s)] \exp \left(-s^{2} / 4\right) d s,
$$

where $a(s) \rightarrow 0$ as $s \rightarrow \infty$. Thus, from

$$
\lim _{y \rightarrow \infty} y^{\delta}\left[\frac{y w^{\prime}(y)}{w(y)}-2 \gamma\right]=0
$$

for $\delta \in(0,2)$, we conclude that for any $\delta \in(0,2)$ there exists a positive constant $C_{\delta}$ such that

$$
w(y)=C_{\delta} y^{2 \gamma}\left[1+o\left(y^{-\delta}\right)\right]
$$

as $y \rightarrow \infty$. The case for $\alpha=\infty$ is similar.

The asymptotic behavior of $w(y)$ at $y=-\infty$ follows by a similar argument and the theorem is proved.

In the sequel, we shall call a nonconstant positive global solution of (1.5), which behaves as $|y|^{2 \gamma}$ both at $y=\infty$ and at $y=-\infty$, as a slow orbit.

REMARK 2.5. For any $\beta>0$, using the Sturm comparison theorem (cf. [10]), we can show that every nonconstant positive solution of (1.5) must be strictly convex for all $y$ sufficiently large and/or for all $-y$ sufficiently large. Hence there is no slow orbit for $\beta \geq 1$.

The proof of Remark 2.5 is quite similar to that of [10, Theorem 2]. Here we compare the function $w^{\prime \prime}$ with the function $w^{-\beta}-w^{\prime \prime}$ and compare the function $w^{-\beta}-w^{\prime \prime}$ with $w^{\prime}$. Then, for a nonconstant positive solution $w(y)$ of (1.5), if $y_{0} \geq 0$ (which we may assume without loss of generality) is such that $w^{\prime}\left(y_{0}\right)=$ and $w^{\prime}(y)>0$ for all $y>y_{0}$, we have $w^{\prime \prime}(y)>0$ for all $y \geq y_{0}$. 
For $0<\beta<1$, let

$$
\phi(y)=K|y|^{2 \gamma}, \quad K=[2 \gamma(2 \gamma-1)]^{-\gamma} .
$$

Note that $K$ is positive, since $\gamma>\frac{1}{2}$ in this case. Also, $\phi(y)$ is a continuously differentiable function satisfying (1.5) for all $y \neq 0$. Similar to [9, Theorem 3.4], we have the following result.

THEOREM 2.6. Any slow orbit $w(y)$ must intersect the function $\phi(y)$ at least twice in $y>0$ and/or in $y<0$.

Proof. Since the proof is quite similar to that of [9, Theorem 3.4], we only sketch the proof. To begin with, we take the minimum point $y_{0}$ of $w$. Without loss of generality we may assume that $y_{0} \geq 0$. First, let

$$
g(y)=\gamma w(y)-\frac{1}{2} y w^{\prime}(y), \quad h(y)=w^{\prime \prime}(y), \quad \text { and } \quad V(y)=g(y) h^{\prime}(y)-g^{\prime}(y) h(y) .
$$

Then

$$
V(y)=\rho^{-1}(y)\left\{\rho\left(y_{0}\right) V\left(y_{0}\right)+\int_{y_{0}}^{y} \rho(t) \beta(\beta+1) w^{-(\beta+2)}(t)\left[w^{\prime}(t)\right]^{2} g(t) d t\right\}
$$

where $\rho(y)=\exp \left(-y^{2} / 4\right)$, and

$$
h(y)=\frac{h\left(y_{0}\right)}{g\left(y_{0}\right)} g(y)+g(y) \int_{y_{0}}^{y} \frac{V(t)}{g^{2}(t)} d t .
$$

Recall that $g(y) \rightarrow 0$ and $g^{\prime}(y) \rightarrow 0$ as $y \rightarrow \infty$ for any slow orbit $w(y)$. By the choice of $y_{0}$, we have $V\left(y_{0}\right) \geq 0$ and $g\left(y_{0}\right)>0$. If $g(y)>0$ for all $y \geq y_{0}$, then we will have $V(y) \rightarrow \infty$ as $y \rightarrow \infty$. Hence $h(y) \rightarrow \infty$ as $y \rightarrow \infty$, a contradiction. Therefore, $g(y)$ must have a zero in $y>y_{0}$.

Next, let

$$
U(y)=w(y) \phi^{\prime}(y)-w^{\prime}(y) \phi(y) .
$$

Then $U(0)=0$ and $U$ satisfies

$$
\begin{aligned}
& U(y)=2 K y^{2 \gamma-1} g(y), \\
& U^{\prime}-\frac{y}{2} U=w \phi\left[\phi^{-(\beta+1)}-w^{-(\beta+1)}\right], \quad y \neq 0 .
\end{aligned}
$$

If $w\left(y_{0}\right)<\phi\left(y_{0}\right)$, then clearly $w$ intersects $\phi$ at least once in $\left(0, y_{0}\right)$. From here and proceeding as in the proof of Lemma 3.6 of [9], we obtain that $w$ intersects $\phi$ at least twice in $y>0$. Hence the theorem follows.

§3. The quenching rate. We assume that $\beta \in(0,1)$ and hence $2 \gamma>1$. Let $a$ be a quenching point for $u$. Without loss of generality we may assume that $a=0$. First, we recall that $w(y, s) \geq 1 / B$ in $W$ for some positive constant $B$. Applying the maximum principle (cf. [6]) to the function

$$
J(x, t)=\frac{1}{2} u_{x}^{2}-\frac{C}{1-\beta}(1-u)^{1-\beta}
$$

for some constant $C \geq 1$, we obtain that

$$
\left|u_{x}\right| \leq \sqrt{\frac{C}{1-\beta}}(1-u)^{(1-\beta) / 2} \quad \text { in }(-l, l) \times(0, T) .
$$


From (3.1) it follows that

$$
w(y, s) \leq C\left(|y|^{2 \gamma}+1\right) \text { in } W .
$$

Now, applying the energy method of [7] (for details see [8]), we can show that $w(y, s)$ tends to a positive global solution of (1.5) as $s \rightarrow \infty$. By (3.2), this limit function must be a slow orbit, if it is not identically equal to the constant $k$.

We claim that this limit function intersects $\phi(y)$ at most once both in $y>0$ and in $y<0$. First, consider the case $y>0$. If $w(y, s)>\phi(y)$ in $W \cap\{y>0\}$, then we are done. Otherwise, we choose $s_{0}<\infty$ such that $w\left(y_{0}, s_{0}\right) \leq \phi\left(y_{0}\right)$ for some $y_{0}>0$ with $\left(y_{0}, s_{0}\right) \in W$. Then proceeding as in the proofs of [1, Sec. 3] there is a $\delta>0$ such that $w(y, s)$ intersects $\phi(y)$ exactly once in $(0, \delta \exp (s / 2))$ for all $s>s_{0}$. The case for $y<0$ is similar. Therefore, the assertion follows. By Theorem 2.6 , this limit function must be identically equal to the constant $k$. Hence Theorem A is proved for $0<\beta<1$.

Acknowledgment. This work was partially supported by the National Science Council of the Republic of China. The author thanks the referee for the useful suggestions on the presentation of this article.

\section{REFERENCES}

[1] J. Bebernes and D. Eberly, A description of self-similar blow-up for dimensions $n \geq 3$, Ann. Inst. Henri Poincaré 5, 1-21 (1988)

[2] H. Brezis, L. A. Peletier, and D. Terman, $A$ very singular solution of the heat equation with absorption, Arch. Rational Mech. Anal. 95, 185-209 (1986)

[3] M. Fila and J. Hulshof, A note on the quenching rate, Proc. Amer. Math. Soc., to appear

[4] M. Fila, J. Hulshof, and P. Quittner, The quenching problem on $N$-dimensional ball, preprint

[5] A. Friedman, J. Friedman, and B. McLeod, Concavity of solutions of nonlinear ordinary differential equations, J. Math. Anal. Appl. 131, 486-500 (1988)

[6] A. Friedman and B. McLeod, Blow-up of positive solutions of semilinear heat equations, Indiana Univ. Math. J. 34, 425-447 (1985)

[7] Y. Giga and R. V. Kohn, Asymptotically self-similar blow-up of semilinear heat equations, Comm. Pure Appl. Math. 38, 297-319 (1985)

[8] J. Guo, On the quenching behavior of the solution of a semilinear parabolic equation, J. Math. Anal. Appl. 151, 58-79 (1990)

[9] J. Guo, On the semilinear elliptic equation $\Delta w-\frac{1}{2} y \cdot \nabla w+\lambda w-w^{-\beta}=0$ in $\mathbf{R}^{n}$, IMA Preprint Series \# 531 (June 1989), Minnesota

[10] H. G. Kaper and M. K. Kwong, Concavity and monotonicity properties of solutions of Emden-Fowler equations, Differential Integral Equations 1, 327-340 (1988)

[11] H. A. Levine, Advances in quenching, Proceedings of the International Conference on Reaction-Diffusion Equations and Their Equilibrium States, Gregynog, Wales, August 1989 